Power Electronics 


\section{Other Macmillan titles of related interest}

W. A. Atherton, From Compass to Computer

B. R. Bannister and D. G. Whitehead, Fundamentals of Modern Digital Systems

G. B. Clayton, Data Converters

G. B. Clayton, Experiments with Operational Amplifiers

J. C. Cluley, Transducers for Microprocessor Systems

R. F. W. Coates, Modern Communication Systems, second edition

A. R. Daniels, Introduction to Electrical Machines

C. W. Davidson, Transmission Lines for Communication

J. D. Edwards, Electrical Machines - An Introduction to Principles and Characteristics

M. Goodge, Semiconductor Device Technology

B. A. Gregory, An Introduction to Electrical Instrumentation and Measurement Systems, second edition

Paul A. Lynn, An Introduction to the Analysis and Processing of Signals, second edition

Paul A. Lynn, Electronic Signals and Systems

S. A. Marshall, Introduction to Control Theory

A. G. Martin and F. W. Stephenson, Linear Microelectronic Systems

J. E. Parton, S. J. T. Owen and M. S. Raven, Applied Electromagnetics, second edition

M. Ramamoorty, An Introduction to Thyristors and their Applications

Douglas A. Ross, Optoelectronic Devices and Optical Imaging Techniques

Trevor J. Terrell, Introduction to Digital Filters

M. J. Usher, Sensors and Transducers

G. Williams, An Introduction to Electrical Circuit Theory 


\title{
Power Electronics
}

Devices, Drivers and Applications

\author{
B.W. Williams \\ B.Sc., Dip.Eng., B.Eng., M.Sc., Ph.D., D.I.C. \\ Professor of Electrical Engineering \\ Department of Electrical and Electronic Engineering \\ Heriot-Watt University, Edinburgh
}

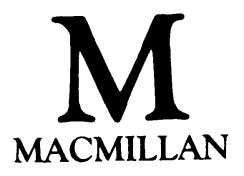


All rights reserved. No reproduction, copy or transmission of this publication may be made without written permission.

No paragraph of this publication may be reproduced, copied or transmitted save with written permission or in accordance with the provisions of the Copyright Act 1956 (as amended).

Any person who does any unauthorised act in relation to this publication may be liable to criminal prosecution and civil claims for damages.

First published 1987

Published by

MACMILLAN EDUCATION LTD

Houndmills, Basingstoke, Hampshire RG21 2XS

and London

Companies and representatives

throughout the world

British Library Cataloguing in Publication Data

Williams, B. W.

Power electronics: devices, drivers and

applications.

1. Power semiconductors

I. Title

$621.3815^{\prime} 2 \quad$ TK7871.85

ISBN 978-0-333-39662-9 ISBN 978-1-349-18525-2 (eBook)

DOI 10.1007/978-1-349-18525-2 


\section{Contents}

Preface

Acknowledgements

List of Symbols

viii

ix

$x$

PART 1: DEVICES 1

1 Basic Semiconductor Physics and Technology 3

1.1 Processes forming pn junctions 4

$\begin{array}{ll}1.2 \text { The masking process } & 6\end{array}$

2 The pn Junction $\quad 8$

2.1 The pn junction under forward bias (steady state) 8

2.2 The pn junction under reverse bias (steady state) 9

$\begin{array}{ll}2.3 \text { Thermal effects } & 12\end{array}$

$\begin{array}{ll}2.4 \text { Piecewise-linear junction diode model } & 13\end{array}$

3 Power Switching Devices and their Static Electrical Characteristics 16

$\begin{array}{lll}3.1 & \text { The power diode } & 16\end{array}$

3.2 Power switching semiconductor transistors 23

3.3 The thyristor $\quad 36$

3.4 The field-controlled thyristor (fct) 50

4 Electrical Ratings and Characteristics of Power Semiconductor Switching Devices $\quad 53$

4.1 General maximum ratings of power semiconductor devices 53

4.2 The fast-recovery diode $\quad 55$

4.3 The bipolar, high-voltage, power switching npn transistor 59

$\begin{array}{ll}4.4 \text { The power mosfet } & 70\end{array}$

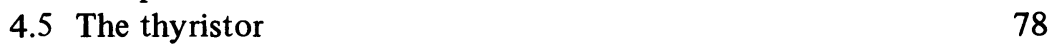


5 Cooling of Power Switching Semiconductor Devices 90

$\begin{array}{lll}5.1 & \text { Thermal resistances } & 91\end{array}$

$\begin{array}{ll}5.2 \text { Modes of power dissipation } & 93\end{array}$

$\begin{array}{ll}5.3 \text { Average power dissipation } & 98\end{array}$

5.4 Power losses from manufacturers' data sheets 98

$\begin{array}{ll}5.5 \text { Heat-sinking design cases } & 100\end{array}$

$\begin{array}{ll}\text { PART 2: DRIVERS } & 111\end{array}$

6 Load Considerations $\quad 113$

$\begin{array}{ll}6.1 \text { The resistive load } & 113\end{array}$

$\begin{array}{ll}6.2 \text { The inductive load } & 116\end{array}$

7 Driving the Transistor, and its Protection 124

$\begin{array}{ll}7.1 \text { Application of the power mosfet } & 124\end{array}$

7.2 Application of the bipolar transistor 133

$\begin{array}{ll}7.3 \text { Transistor combinations } & 151\end{array}$

7.4 Appendix: Turn-off switching-aid circuit analysis 154

8 Driving the Thyristor, and its Protection $\quad 159$

$\begin{array}{ll}8.1 \text { Gate drive circuits } & 160\end{array}$

$\begin{array}{ll}8.2 \text { Gate drive design } & 162\end{array}$

$\begin{array}{ll}\text { 8.3 Drive design for gto's } & 164\end{array}$

8.4 Thyristor switching-aid circuits 164

8.5 Switching-aid circuit for the gto thyristor 170

8.6 Appendix: Turn-off snubber circuit analysis 170

9 Switching-aid Circuits with Energy Recovery 173

9.1 Energy recovery for turn-on snubber circuits 173

$\begin{array}{ll}\text { 9.2 Energy recovery for turn-off snubber circuits } & 177\end{array}$

9.3 Unified turn-on and turn-off snubber circuit energy recovery 184

10 Series and Parallel Device Operation and Protection 187

10.1 Parallel and series operation of power devices 187

$\begin{array}{ll}10.2 \text { Protection } & 198\end{array}$

$\begin{array}{lr}\text { PART 3: APPLICATIONS } & 213\end{array}$

11 Naturally Commutating Converters 215

11.1 Single-phase uncontrolled converter circuits 216

$\begin{array}{ll}11.2 \text { Single-phase half-controlled converter } & 224\end{array}$ 


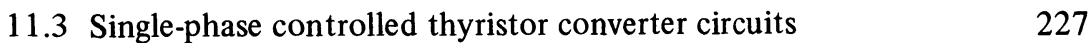

11.4 Three-phase uncontrolled converter circuits 233

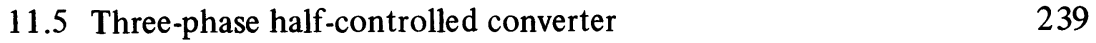

11.6 Three-phase controlled thyristor converter circuits 239

12 AC Voltage Regulators $\quad 249$

$\begin{array}{ll}12.1 \text { Single-phase ac regulator } & 249\end{array}$

$\begin{array}{ll}12.2 \text { Three-phase ac regulator } & 251\end{array}$

$\begin{array}{ll}12.3 \text { Integral cycle control } & 256\end{array}$

$\begin{array}{ll}12.4 \text { Single-phase transformer tap changer } 258 & 256\end{array}$

$\begin{array}{ll}12.5 \text { Cycloconverter } & 259\end{array}$

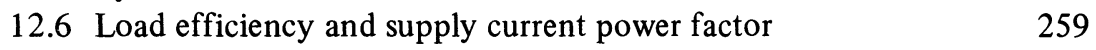

13 Thyristor Forced Commutated DC Choppers 264

13.1 A thyristor reverse impressed voltage commutated dc chopper 266

13.2 A thyristor current impulse displacement commutated dc
chopper

$\begin{array}{ll}\text { 13.3 Other cid and riv commutation circuits } & 277\end{array}$

13.4 Comparison between the cid and riv commutation processes 279

14 Static Power Inverters $\quad 282$

14.1 Voltage-fed inverter bridge topologies 283

14.2 Controlled current-sourced inverters 304

15 Switched-mode Power Supplies 309

15.1 The forward converter 309

$\begin{array}{ll}15.2 \text { The flyback converter } & 314\end{array}$

$\begin{array}{ll}15.3 \text { Comparison of basic converters } & 321\end{array}$

15.4 Multiple-switch, balanced, isolated converters 324

Bibliography $\quad 330$

Index 


\section{Preface}

The objective of this book is to bridge the gap between semiconductor device theory and device application in the area of power electronics. The majority of existing books concentrate on one aspect at the expense of the other. They either analyse semiconductor physics and material aspects of devices without including any applications or, at the other end of the spectrum, they consider device applications with the device being treated as a 'black box'.

This book specifically considers high-power devices and in particular how a device's structure and construction are related to its terminal electrical and thermal characteristics. Only then, armed with an understanding of device characteristics, is it possible to select the correct device for a given application.

Emphasis is placed on the circuitry required to use power devtces rather than treating them as ideal switches or considering just one device type. The structure and electrical characteristics of a device are related to its driving and protection requirements for a particular type of application.

The book is in three parts. Part 1 covers power device electrical and thermal characteristics and how they relate to a device's physical structure. Devices considered include the thyristor, gto, mosfet and bipolar transistor. Part 2 describes device driving and protection techniques and the final part gives a general selection of power electronic applications. Application areas other than those dominated by thyristors have been considered; in particular the growing area of switchedmode power supplies has been introduced.

The book gives a fundamental, yet detailed education in the art of power electronics. Since a basic knowledge of semiconductor physics and circuit techniques is assumed, it is a specialist subject book intended for university and polytechnic students, as well as for practising engineers wishing to broaden their understanding of power electronics. Non-trivial worked examples and problems have been included for the student, and each chapter has a specific reading list.

B. W. W. 


\section{Acknowledgements}

I would like to thank Motorola, International Rectifier and Siemens, who have permitted use of their published material. I am indebted to Elizabeth Boden who has not been content with just typing the manuscript but has attempted to eliminate those errors of grammar and punctuation that I tend to overlook. 


\section{List of Symbols}

a device tolerance

A anode

b base

$b_{t} \quad$ base transport factor

$B_{\text {sat }}$ saturation flux density

c collector

$C$ capacitance

$C_{\mathrm{ds}} \quad$ drain-to-source capacitance

$C_{\mathrm{f}} \quad$ heat-sink correction factor

$C_{\mathrm{gd}}$ gate-to-drain capacitance

$C_{\mathrm{gs}} \quad$ gate-to-source capacitance

$C_{\mathrm{o}} \quad$ snubber transfer capacitance

$C_{\mathrm{s}} \quad$ snubber capacitance

d drain

$e_{\mathrm{o}} \quad$ output voltage

$e_{\mathrm{s}} \quad$ step input voltage

$E \quad$ circuit voltage source

$E_{\mathrm{i}} \quad$ input voltage source

ESR equivalent series resistance

$f_{\mathrm{m}} \quad$ maximum switching frequency

$f_{\mathrm{s}} \quad$ switching frequency

g mosfet gate

G thyristor gate 
$h \quad$ heat transfer coefficient

$H_{\mathrm{s}} \quad$ flux intensity at saturation

$i \quad$ current

$i_{\mathrm{b}} \quad$ base current

$i_{\mathbf{i}} \quad$ input currrent

$i_{1} \quad$ inductor current

$i_{\mathrm{o}} \quad$ output current

$i_{\mathrm{rr}} \quad$ reverse recovery current

$i_{\mathrm{T}} \quad$ thyristor anode current

$I_{\mathrm{a}} \quad$ prospective fault current

$I_{\mathrm{c}} \quad$ collector current

$I_{\mathrm{cbo}} \quad$ collector leakage current

$I_{\mathrm{c}(\max )}$ maximum allowable collector current

$I_{\mathrm{d}} \quad$ drain current

$I_{\mathrm{d}(\mathrm{rms})}$ rms drain current

$I_{\mathrm{D}} \quad$ mean diode current

$I_{\mathrm{F}} \quad$ forward current

$I_{\mathrm{F} \text { (av) }}$ average forward current

$I_{\mathrm{G}} \quad$ gate current

$I_{\mathrm{H}}$ holding current

$\bar{I}_{\mathbf{i}} \quad$ mean input current

$I_{1} \quad$ leakage current

$I_{\mathrm{L}} \quad$ latching current

$I_{\mathrm{O}} \quad$ reverse leakage current

$\bar{I}_{\mathbf{O}} \quad$ mean output current

$I_{\mathrm{p}}$ peak let-through current

$I_{\mathrm{rr}}$ peak reverse recovery current

$I_{\mathrm{s} / \mathrm{b}} \quad$ second breakdown current

$I_{\mathrm{T}} \quad$ total current

J junction

$J_{\mathrm{R}} \quad$ reverse recovery energy

$k \quad$ transistor switching time ratio

K cathode

$K_{\text {ID }}$ diode current form factor

$K_{\mathrm{v}} \quad$ voltage ripple factor

$l_{\mathrm{p}} \quad$ primary inductance

$l_{\mathrm{s}} \quad$ saturable inductance

$L \quad$ inductance

$L_{\mathrm{s}} \quad$ linear snubber inductance 


\begin{tabular}{|c|c|}
\hline xii & List of Symbols \\
\hline$m$ & empirical transistor constant \\
\hline$M$ & avalanche multiplication factor \\
\hline$n$ & ratio $C_{\mathrm{s}} / C_{\mathrm{o}}$ \\
\hline$n_{\mathbf{i}}$ & intrinsic carrier concentration \\
\hline$N$ & turns or turns ratio \\
\hline$N_{\mathrm{A}}$ & acceptor concentration \\
\hline$N_{\mathrm{C}}$ & concentration \\
\hline$N_{\mathrm{D}}$ & donor concentration \\
\hline$p d$ & percentage derating \\
\hline$P$ & power \\
\hline$P_{\mathrm{d}}$ & average power dissipation \\
\hline$P_{\mathrm{G}}$ & mean gate power \\
\hline$P_{\mathrm{p}}$ & peak power \\
\hline$P_{\mathrm{s}}$ & switching interval power loss \\
\hline$Q_{\mathrm{G}}$ & mosfet gate charge \\
\hline$Q_{\mathrm{o}}$ & diode total recovery charge \\
\hline$Q_{\mathrm{R}}$ & diode reverse recovery charge \\
\hline$r_{\mathrm{b}}^{\prime}$ & base lateral resistance \\
\hline$r\left(t_{\mathrm{p}}\right)$ & thermal normalising factor \\
\hline$R_{\mathrm{be}}$ & base-to-emitter resistance \\
\hline$R_{\mathrm{ds} \text { (on) }}$ & mosfet on-state resistance \\
\hline$R_{\mathrm{g}}$ & gate resistance \\
\hline$R_{\mathrm{L}}$ & load resistance \\
\hline$R_{\theta}$ & thermal resistance \\
\hline$R_{\theta \mathrm{j}-\mathrm{c}}$ & thermal resistance junction-to-case \\
\hline s & source \\
\hline$S$ & $\operatorname{maximum} \mathrm{d} v / \mathrm{d} t$ \\
\hline SOA & safe operating area \\
\hline$t$ & time \\
\hline$t_{\mathrm{a}}$ & fuse arcing time \\
\hline$t_{\mathrm{c}}$ & fuse clearing time \\
\hline$t_{\mathrm{d}}$ & turn-on delay time \\
\hline$t_{\mathrm{D}}$ & diode conduction time \\
\hline$t_{\mathrm{fi}}$ & current fall time \\
\hline$t_{\mathrm{fr}}$ & diode turn-on time \\
\hline$t_{\mathrm{fv}}$ & voltage fall time \\
\hline$t_{\mathrm{m}}$ & fuse melting time \\
\hline$t_{\mathrm{off}}$ & turn-off time \\
\hline
\end{tabular}




$\begin{array}{ll}t_{\mathrm{on}} & \text { turn-on time } \\ t_{\mathrm{p}} & \text { pulse width } \\ t_{\mathrm{q}} & \text { thyristor turn-off time } \\ t_{\mathrm{ri}} & \text { current rise time } \\ t_{\mathrm{rr}} & \text { reverse recovery time } \\ t_{\mathrm{s}} & \text { saturation delay time } \\ t_{\mathrm{T}} & \text { transistor on-time } \\ t_{\mathrm{x}} & \text { time of no conduction } \\ T & \text { temperature } \\ T_{\mathrm{a}} & \text { ambient temperature } \\ T_{\mathrm{c}} & \text { case temperature } \\ T_{\mathrm{j}} & \text { junction temperature } \\ T_{\mathrm{jmax}} & \text { maximum allowable junction temperature } \\ T_{\mathrm{m}} & \text { main thyristor }\end{array}$

$\begin{array}{ll}v & \text { voltage } \\ v_{\mathrm{be}(\mathrm{sat})} & \text { base-to-emitter saturation voltage } \\ v_{\mathrm{ce}(\mathrm{sat})} & \text { collector-to-emitter saturation voltage } \\ v_{\mathrm{D}} & \text { diode on-state voltage } \\ v_{\mathrm{o}} & \text { output voltage } \\ V & \text { voltage } \\ V_{\mathrm{a}} & \text { common emitter avalanche voltage } \\ V_{\mathrm{ac}} & \text { ac supply voltage } \\ V_{\mathrm{b}} & \text { junction avalanche voltage } \\ V_{\mathrm{be}} & \text { base-to-emitter voltage } \\ V_{\mathrm{BF}} & \text { scr forward breakover voltage } \\ V_{\mathrm{BR}} & \text { scr reverse breakdown voltage } \\ V_{\mathrm{c}} & \text { collector voltage } \\ V_{\mathrm{ce}} & \text { collector-to-emitter voltage } \\ V_{\mathrm{D}} & \text { device voltage rating } \\ V_{\mathrm{ds}} & \text { drain-to-source voltage } \\ V_{\mathrm{fp}} & \text { diode turn-on peak forward voltage } \\ V_{\mathrm{gs}} & \text { gate-to-source voltage } \\ V_{\mathrm{L}} & \text { line-to-line rms voltage } \\ V_{\mathrm{o}} & \text { mean output voltage } \\ V_{\mathrm{PT}} & \text { punch-through voltage } \\ V_{\mathrm{r}} & \text { rms output voltage } \\ V_{\mathrm{R}} & \text { reverse applied voltage } \\ V_{\mathrm{RI}} & \text { ripple voltage } \\ V_{\mathrm{R} \mathrm{M}} & \text { reverse overshoot voltage } \\ V_{\mathrm{s}} & \text { dc supply voltage } \\ V_{\mathrm{TH}} & \text { mosfet threshold voltage } \\ V_{\mathrm{Z}} & \text { Zener voltage } \\ & \end{array}$




$\begin{array}{ll}\text { xiv } & \\ w & \text { thickness } \\ W & \text { energy } \\ W_{\mathrm{t}} & \text { total loss } \\ Z & \text { impedance } \\ Z_{\theta} & \text { thermal impedance } \\ & \\ \alpha & \text { current transfer ratio } \\ \alpha_{\mathrm{o}} & \alpha \text { in the linear region } \\ \beta & \text { current amplification factor } \\ \beta_{\mathrm{Q}} & \text { gto turn-off gain } \\ \gamma & \text { injection efficiency } \\ \delta & \text { duty cycle } \\ \Delta T & \text { change in temperature } \\ \lambda & \text { thermal conductance } \\ \xi & \text { damping factor } \\ \xi_{\mathrm{m}} & \text { peak electric field } \\ \sigma & \text { electrical conductivity } \\ \tau & \text { period } \\ \phi & \text { tan }{ }^{-1} \text { ( } \omega L / R \text { ) } \\ \Phi & \text { zero bias potential } \\ \omega & \text { oscillation frequency } \\ \omega_{\mathrm{o}} & \text { natural oscillation frequency } \\ & \\ \vee & \text { peak } \\ & \text { minimum } \\ & \end{array}$

